\title{
Half-positional Determinacy of Infinite Games
}

\author{
Eryk Kopczyński ${ }^{\star}$ \\ Institute of Informatics, Warsaw University \\ erykk@mimuw.edu.pl
}

\begin{abstract}
We study infinite games where one of the players always has a positional (memory-less) winning strategy, while the other player may use a history-dependent strategy. We investigate winning conditions which guarantee such a property for all arenas, or all finite arenas. We establish some closure properties of such conditions, and discover some common reasons behind several known and new positional determinacy results. We exhibit several new classes of winning conditions having this property: the class of concave conditions (for finite arenas) and the classes of monotonic conditions and geometrical conditions (for all arenas).
\end{abstract}

\section{Introduction}

The theory of infinite games is relevant for computer science because of its potential application to verification of interactive systems. In this approach, the system and environment are modeled as players in an infinite game played on a graph (called arena) whose vertices represent possible system states. The players (conventionally called Eve and Adam) decide which edge (state transition, or move) to choose; each edge has a specific color. The desired system's behavior is expressed as a winning condition of the game - the winner depends on the sequence of colors which appear during an infinite play. If a winning strategy exists in this game, the system which implements it will behave as expected. Positional strategies (i.e. depending only on the position, not on the history of play - also called memoryless) are of special interest here, because of their good algorithmic properties which can lead to an efficient implementation.

Among the most often used winning conditions are the parity conditions, which admit positional determinacy ([Mos91], [EJ91], [McN93]). However, not always it is possible to express the desired behavior as a parity condition. An interesting question is, what properties are enough for the winning condition to be positionally determined, i.e. admit positional winning strategies independently on the arena on which the game is played. Recently some interesting characterizations of such positionally determined winning conditions have been found. In [CN06] it has been proven that every (prefix independent) condition which admits positional determinacy for all finite and infinite arenas (with colored moves) is a parity condition (up to renaming colors). There are more such conditions if we only consider finite arenas. In [GZ05] it has been proven that a winning condition is positionally determined for all finite arenas whenever it is so for finite

\footnotetext{
* Supported by KBN grant 4 T11C 04225 and the EC RTN GAMES.
} 
arenas where only one player is active. Another interesting characterization can be found in [GZ04]. For a survey of recent results on positional determinacy see [Gra04].

Our work attempts to obtain similar characterizations and find interesting properties (e.g. closure properties) of half-positionally determined winning conditions, i.e. ones such that all games using such a winning condition are positionally determined for one of the players (us, say), but the other player (environment) can have an arbitrary strategy. We give uniform arguments to prove several known and several new half-positional determinacy results. As we will see, some results on positional determinacy have natural generalizations to half-positional determinacy, but some do not. This makes the theory of half-positional conditions harder than the theory of positional conditions.

We also exhibit some large classes of half-positionally determined winning conditions. One example is the class of concave winning conditions; among examples of such conditions are the parity conditions, Rabin conditions, and the geometrical condition associated with convex subsets of $[0,1]^{n}$. Concavity is sufficient for half-positional determinacy only in the case of games on finite arenas. We investigate to what extent the results on geometrical conditions can be extended to infinite arenas. Another example is the class of monotonic winning conditions, which are defined using a deterministic finite automaton with a monotonic transition function, and includes winning conditions such as $C^{\omega}-C^{*}\left(a^{n} C^{*}\right)^{\omega}$. Monotonic winning conditions are half-positionally determined on all arenas.

Due to space limitations we had to omit most of proofs and algorithms. They will be presented in the full version of this paper. Its draft can be found at $[\mathrm{Kop} 06]$.

\section{Preliminaries}

We consider perfect information antagonistic infinite games played by two players, called conventionally Adam and Eve. Let $C$ be a set of colors (possibly infinite).

An arena over $C$ is a tuple $G=\left(\operatorname{Pos}_{A}, \operatorname{Pos}_{E}\right.$, Mov $)$, where:

- Elements of Pos $=\operatorname{Pos}_{E} \cup \operatorname{Pos}_{A}$ are called positions; $\operatorname{Pos}_{A}$ and $\operatorname{Pos}_{E}$ are disjoint sets of Adam's positions and Eve's positions, respectively.

- Elements of Mov $\subseteq \operatorname{Pos} \times \operatorname{Pos} \times C$ are called moves; $\left(v_{1}, v_{2}, c\right)$ is a move from $v_{1}$ to $v_{2}$ colored by $c$. We denote $\operatorname{source}\left(v_{1}, v_{2}, c\right)=v_{1}, \operatorname{target}\left(v_{1}, v_{2}, c\right)=v_{2}$, $\operatorname{rank}\left(v_{1}, v_{2}, c\right)=c$.

A game is a pair $(G, W)$, where $\mathrm{G}$ is an arena, and $\mathrm{W}$ is a winning condition. A winning condition $W$ over $C$ is a subset of $C^{\omega}$ which is prefix independent, i.e., $u \in W \Longleftrightarrow c u \in W$ for each $c \in C, u \in C^{\omega}$. We name specific winning conditions $W A, W B, \ldots$ For example, the parity condition of rank $n$ is the winning condition over $C=\{0,1, \ldots, n\}$ defined with

$$
W P_{n}=\left\{w \in C^{\omega}: \limsup _{i \rightarrow \infty} w_{i} \text { is even }\right\}
$$


The game $(G, W)$ carries on in the following way. The play starts in some position $v_{1}$. The owner of $v_{1}$ (e.g. Eve if $v_{1} \in \operatorname{Pos}_{E}$ ) chooses one of the moves leaving $v_{1}$, say $\left(v_{1}, v_{2}, c_{1}\right)$. If the player cannot choose because there are no moves leaving $v_{1}$, he or she loses. The next move is chosen by the owner of $v_{2}$; denote it by $\left(v_{2}, v_{3}, c_{2}\right)$. And so on: in the $n$-th move the owner of $v_{n}$ chooses a move $\left(v_{n}, v_{n+1}, c_{n}\right)$. If $c_{1} c_{2} c_{3} \ldots \in W$, Eve wins the infinite play; otherwise Adam wins.

A play in the arena $G$ is any sequence of moves $\pi$ such that $\operatorname{source}\left(\pi_{n+1}\right)=$ $\operatorname{target}\left(\pi_{n}\right)$. By $\operatorname{source}(\pi)$ and $\operatorname{target}(\pi)$ we denote the initial and final position of the play, respectively. The play can be finite $\left(\pi \in \operatorname{Pos} \cup \mathrm{Mov}^{+}\right.$, where by $\pi \in \operatorname{Pos}$ we represent the play which has just started in the position $\pi$ ) or infinite $\left(\pi \in \mathrm{Mov}^{\omega}\right.$; infinite plays have no target).

A strategy for player $X$ is a partial function $s: \operatorname{Pos} \cup \mathrm{Mov}^{+} \rightarrow$ Mov. For a finite play $\pi$ such that $\operatorname{target}(\pi) \in \operatorname{Pos}_{X}, s(\pi)$ says what $X$ should do in the next move. A strategy $s$ is winning (for $X$ ) from the position $v$ if $s(\pi)$ is defined for each finite play $\pi$ starting in $v$, consistent with $s$, and ending in $\operatorname{Pos}_{X}$, and each infinite play starting in $v$ consistent with $s$ is winning for $X$.

A strategy $s$ is positional if it depends only on $\operatorname{target}(\pi)$, i.e., for each finite play $\pi$ we have $s(\pi)=s(\operatorname{target}(\pi))$.

A game is determined if for each starting position one of the players has a winning strategy. This player may depend on the starting position in the given play. Thus if the game is determined, the set Pos can be split into two sets $\mathrm{Win}^{E}$ and $\mathrm{Win}^{A}$ and there exist strategies $s_{E}$ and $s_{A}$ such that each play $\pi$ with $\operatorname{source}(\pi) \in \mathrm{Win}^{X}$ and consistent with $s_{X}$ is winning for $X$. All games with a Borel winning condition are determined [Mar75], but there exist (exotic) games which are not determined. A winning condition $W$ is determined if for each arena $G$ the game $(G, W)$ is determined.

We are interested in games and winning conditions for which one or both of the players have positional winning strategies. Thus, we introduce the notion of a determinacy type, given by three parameters: admissible strategies for Eve (positional or arbitrary), admissible strategies for Adam (positional or arbitrary), and admissible arenas (finite or infinite). We say that a winning condition $W$ is $(\alpha, \beta, \gamma)$-determined if for every $\gamma$-arena $G$ the game $(G, W)$ is $(\alpha, \beta)$-determined, i.e. for every starting position either Eve has a winning $\alpha$ strategy, or Adam has a winning $\beta$-strategy. Clearly, there are 8 determinacy types in total. For short, we call (positional, positional, infinite)-determined winning conditions positionally determined or just positional, (positional, arbitrary, infinite)-determined winning conditions half-positional, (arbitrary, positional, infinite)-determined winning conditions co-half-positional. If we restrict ourselves to finite arenas, we add finitely, e.g. (positional, arbitrary, finite)-determined conditions are called finitely half-positional. For a determinacy type $D=(\alpha, \beta, \gamma), D$-arenas mean $\gamma$-arenas, and $D$-strategies mean $\alpha$-strategies (if they are strategies for Eve) or $\beta$-strategies (for Adam).

Note that if a game $(G, W)$ is $(\alpha, \beta)$-determined, then its dual game obtained by using the complement winning condition and switching the roles of players is 
$(\beta, \alpha)$-determined. Thus, $W$ is $(\alpha, \beta, \gamma)$-determined iff its complement is $(\beta, \alpha, \gamma)$ determined.

In the games defined above the moves are colored. In the literature one often studies similar games where positions are colored instead - in this case instead of Mov $\subseteq \operatorname{Pos} \times \operatorname{Pos} \times C$ we have Mov $\subseteq \operatorname{Pos} \times \operatorname{Pos}$ and a function rank: Pos $\rightarrow C$. The winner of a play in such games is defined similarly.

A position-colored game can be easily transformed into a move-colored game - we just have to color each move $m$ with the color $\operatorname{rank}(\operatorname{target}(m))$. Transformation in the other way in general would require splitting positions when they are targets of moves of different colors, which may cause a previously non-positional strategy to become positional. Hence, for position-colored games there are more (half-)positionally determined winning conditions than for movecolored games. The facts proven or cited here do not necessarily hold in the case of position-colored games.

\section{Closure Properties of Half-positional Conditions}

Now we will give some closure properties of half-positionally determined winning conditions. We will start with a lemma which is used in many proofs of halfpositional determinacy of various winning conditions. This lemma can be proven by transfinite induction.

Lemma 1. Let $D$ be a determinacy type. Let $W \subseteq C^{\omega}$ be a winning condition. Suppose that, for each non-empty $D$-arena $G$ over $C$, there exists a non-empty subset $M \subseteq G$ such that in game $(G, W)$ one of the players has a D-strategy winning from $M$. Then $W$ is $D$-determined.

Definition 1. For $S \subseteq C, W B_{S}$ is the set of infinite words where elements of $S$ occur infinitely often, i.e. $\left(C^{*} S\right)^{\omega}$. Winning conditions of this form are called Büchi conditions. Complements of Büchi conditons, $W B_{S}^{\prime}=C^{*}(C-S)^{\omega}$ are called co-Büchi conditions.

Theorem 1. Let $D$ be a determinacy type. Let $W \subseteq C^{\omega}$ be a winning condition, and $S \subseteq C$. If $W$ is $D$-determined, then so is $W \cup W B_{S}$.

Proof. We will show that the assumption of Lemma 1 holds. Let our arena be $G=\left(\operatorname{Pos}_{E}, \operatorname{Pos}_{A}, \mathrm{Mov}\right) . S$-moves are moves $m$ such that $\operatorname{rank}(m) \in S$.

Let $G^{\prime}$ be $G$ with a new position $T$ added. The position $T$ belongs to Adam and has no outgoing moves, hence Adam loses here. For each $S$-move $m$ we change target $(m)$ to $T$.

Since Adam immediately loses after doing an $S$-move in $G^{\prime}$, the winning conditions $W$ and $W \cup W B_{S}$ are equivalent for $G^{\prime}$, thus we can use $D$-determinacy of $W$ to find the winning sets $\mathrm{Win}_{E}^{\prime}, \mathrm{Win}_{A}^{\prime}$ and winning $D$-strategies $s_{E}^{\prime}, s_{A}^{\prime}$ in $G^{\prime}$.

Suppose $\mathrm{Win}_{A}^{\prime} \neq \emptyset$. We can see that since Adam's strategy wins in $G^{\prime}$ from a starting position in $\mathrm{Win}_{A}^{\prime}$, he also wins in $G$ from there by using the same 
strategy (the game $G^{\prime}$ is ,harder" for Adam than $G$ ). Thus the assumption of 1 holds (we take $M=\mathrm{Win}_{A}^{\prime}$ ).

Now suppose that $\operatorname{Win}_{A}^{\prime}=\emptyset$. We will show that Eve has a winning $D$ strategy $s$ in Pos everywhere, hence the assumption of Lemma 1 also holds (we take $M=$ Win $_{E}^{\prime}$ ).

The strategy is as follows. For a finite play $\pi$ we take $s(\pi)=s_{E}\left(\pi^{\prime}\right)$, where $\pi^{\prime}$ is the longest final segment without any $S$-moves. If $s_{E}$ tells Eve to make an $S$-move, Eve makes its counterpart (or one of its counterparts) in $G$ instead. The strategy $s$ is positional if $s_{E}$ is positional. It can be easily shown that $s$ is indeed a winning strategy.

Note that, by duality, Thm 1 implies that if $W$ is $D$-determined, then so is $W \cap W B_{S}^{\prime}$. This yields an easy proof of positional determinacy of parity conditions. It is enough to start with an empty winning condition (which is positionally determined) and apply Thm 1 and its dual $n$ times.

A union of co-Büchi and co-half-positional conditions does not need to be co-half-positional ( $W B_{\{a\}}^{\prime} \cup W B_{\{b\}}^{\prime}$ is not). What about a union of co-Büchi and a half-positional condition, does it have to be half-positional? We have no proof nor counterexample for this yet. This conjecture can be generalized to the following:

Conjecture 1. Let $\mathcal{W}$ be a (finite, countable, ...) family of half-positionally (finitely) determined winning conditions. Then $\bigcup \mathcal{W}$ is a half-positionally (finitely) determined winning condition.

Note that we assume prefix independence here. It is very easy to find two prefix dependent winning conditions which are positionally determined, but their union is not half-positionally determined.

This conjecture also fails for non-countable families and infinite arenas, even for such simple conditions as Büchi and co-Büchi conditions:

Theorem 2. There exists a family of $2^{\omega}$ Büchi (co-Büchi) conditions such that its union is not a half-positionally determined winning condition.

Proof. Let $I=\omega^{\omega}$. Our arena $A$ consists of one Eve's position $E$ and infinitely many Adam's positions $\left(A_{n}\right)_{n \in \omega}$. In $E$ Eve can choose $n \in \omega$ and go to $A_{n}$ by move $\left(E, A_{n}, \star\right)$. In each $A_{n}$ Adam can choose $r \in \omega$ and return to $E$ by move $\left(A_{n}, E,(n, r)\right)$.

For each $y \in I$, let $S_{y}=\left\{\left(n, y_{n}\right): n \in \omega\right\} \subseteq C$, and $S_{y}^{\prime}=C-S_{y}-\{\star\}$. Let $W A_{1}=\bigcup_{y \in I} W B_{S_{y}}, W A_{2}=\bigcup_{y \in I} W B_{S_{y}^{\prime}}^{\prime}$.

The games $\left(A, W A_{1}\right)$ and $\left(A, W A_{2}\right)$ are not half-positionally determined. Let $\left(n_{k}\right)$ and $\left(r_{k}\right)$ be $n$ and $r$ chosen by Eve and Adam in the $k$-th round, respectively. If Eve always plays $n_{k}=k$, she will win both the conditions $W B_{S_{y}}$ and $W B_{S_{y}^{\prime}}^{\prime}$, where $y_{k}=r_{k}$. However, if Eve plays with a positional strategy $n_{k}=n$, Adam can win by playing $r_{k}=k$.

There is however a subclass of half-positional winning conditions for which we can prove that it is closed under countable union. 
Definition 2. A suspendable winning strategy for $X$ is a pair $(s, \Sigma)$, where $s: \mathrm{Pos} \cup \mathrm{Mov}^{+} \rightarrow \mathrm{Mov}$ is a strategy, and $\Sigma \subseteq \mathrm{Mov}^{*}$, such that:

$-s$ is defined for every finite play $\pi$ such that $\operatorname{target}(\pi) \in \operatorname{Pos}_{X}$.

- every infinite play $\pi$ that is consistent with $s$ from some point $t^{1}$ has a prefix longer than $t$ which is in $\Sigma$;

- Every infinite play $\pi$ that has infinitely many prefixes in $\Sigma$ is winning for $X$.

We say that $X$ has a suspendable winning strategy in $\mathrm{Win}_{X}$ when he has a suspendable winning strategy in the arena $\left(\operatorname{Pos}_{A} \cap \operatorname{Win}_{X}, \operatorname{Pos}_{E} \cap \operatorname{Win}_{X}, \operatorname{Mov} \cap\right.$ $\left.\operatorname{Win}_{X} \times \operatorname{Win}_{X} \times C\right)$.

A winning condition $W$ is positional/suspendable if for each arena $G$ in the game $(G, W)$ Eve has a positional winning strategy in $\mathrm{Win}_{E}$ and Adam has a suspendable winning strategy in $\mathrm{Win}_{A}$.

Intuitively, if at some moment $X$ decides to play consistently with $s$, the play will eventually reach $\Sigma ; \Sigma$ is the set of moments when $X$ can temporarily suspend using the strategy $s$ and return to it later without a risk of ruining his or her victory.

A suspendable winning strategy is a winning strategy, because the conditions above imply that each play which is always consistent with $s$ has infinitely many prefixes in $\Sigma$, and thus is winning for $X$. The co-Büchi condition is positional/suspendable; more examples will be given in Theorems 5 and 6 . However, the parity condition $\mathrm{WP}_{2}$ is positional, but not positional/suspendable, because a suspendable strategy cannot be winning for Adam - it is possible that the play enters state 2 infinitely many times while it is suspended.

Theorem 3. A union of countably many positional/suspendable conditions is also positional/suspendable.

If Adam has a suspendable winning strategy for each of given winning conditions and each starting position, then he can use them all in a play - he just has to activate and suspend each of them infinitely many times. Otherwise, we use a lemma similar to Lemma 1 to remove all positions from where Eve can win.

\section{Concave Winning Conditions}

We will now give some examples of half-positionally determined winning conditions. We will start by giving a simple combinatorial property which guarantees finite half-positional determinacy.

Definition 3. A word $w \in \Sigma^{*} \cup \Sigma^{\omega}$ is a (proper) combination of words $w_{1}$ and $w_{2}$, iff for some sequence of words $\left(u_{n}\right), u_{n} \in \Sigma^{*}$

\footnotetext{
${ }^{1}$ That is, for each prefix $u$ of $\pi$ which is longer than $t$ and such that $\operatorname{target}(u) \in \operatorname{Pos}_{X}$, the next move is given by $s(u)$.
} 


$$
\begin{aligned}
& -w=\prod_{k \in \mathbb{N}} u_{k}=u_{0} u_{1} u_{2} u_{3} u_{4} u_{5} u_{6} u_{7} u_{8} \ldots, \\
& -w_{1}=\prod_{k \in \mathbb{N}} u_{2 k+1}=u_{1} u_{3} u_{5} u_{7} \ldots \\
& -w_{2}=\prod_{k \in \mathbb{N}} u_{2 k}=u_{0} u_{2} u_{4} u_{6} \ldots
\end{aligned}
$$

Definition 4. A winning condition $W$ is convex if as a subset of $C^{\omega}$ it is closed under combinations, and concave if its complement is convex.

Example 1. Parity conditions (including Büchi and co-Büchi conditions) are both convex and concave.

Example 2. Let $C$ be an infinite set. The folowing winning conditions are both convex and concave:

- Exploration condition: the set of all $v$ in $C^{\omega}$ such that $\left\{v_{n}: n \in \omega\right\}$ is infinite.

- Unboundedness condition: the set of all $v$ in $C^{\omega}$ such that no color appears infinitely often.

Decidability and positional determinacy of these conditions on (infinite) pushdown arenas where each position has a distinct color has been studied in [Gim04] (exploration condition) and [BSW03], [CDT02] (unboundedness condition).

Example 3. Concave winning conditions are closed under union. Convex winning conditions are closed under intersection.

Another example (which justifies the name) is given in Section 6 below.

Theorem 4. Concave winning conditions are half-positionally finitely determined.

The proof goes by induction over Mov, and is based on the following idea. Let $v$ be Eve's position, with outgoing moves $m_{1}, m_{2}, \ldots$. Suppose that Eve cannot win by using only one of these moves. Then, since the winning condition is concave, she also cannot win by using many of these moves - because it can be written as a combination of subplays that appear after each move $m_{1}, m_{2}, \ldots$, and Adam wins all of these plays.

This theorem gives yet another proof of finite positional determinacy of parity games, and also half-positional determinacy of unions of families of parity conditions (where each parity condition may use a different rank for a given color). Half-positional determinacy of Rabin conditions (finite unions of families of parity conditions) over infinite arenas has been proven in [Kla92].

Note that concavity does not imply half-positional determinacy over infinite arenas - for examples see Section 6 below, and also Example 2 and Thm 2. Also, half-positional determinacy (even over infinite arenas) does not imply concavity — examples can be found in Sections 6 and 7 .

Concavity does not force any bound on the memory required by Adam. Consider the game $(G, W)$, where $G$ is the arena with one Adam's position $A$ and two moves $A \rightarrow A$ colored 0 and 1 respectively, and $W=W F^{\prime}([0,1]-\{x\})$, 
where $x \in[0,1]$ is irrational. ( $W F^{\prime}$ is defined in Section 6 below.) Adam requires unbounded memory here.

The following proposition gives some algorithmic properties of concavity, assuming that our winning condition is an $\omega$-regular language.

Proposition 1. Suppose that a winning condition $W$ is given by a deterministic parity automaton on infinite words using s states and d ranks. Then there exists a polynomial algorithm of determining whether $W$ is concave (or convex). If $W$ is concave and $G$ is an arena with $n$ positions, then the winning sets and Eve's positional strategy can be found in time $O\left(n(n s)^{d / 2} \log s\right)$.

\section{Weakening the Concavity Condition}

In [GZ04] a result similar to Thm 4 has been obtained in the case of full positional determinacy. To present it, we need the following definition:

Definition 5. A winning condition $W$ is weakly convex iff for each sequence of words $\left(u_{n}\right), u_{n} \in C^{*}$, if

1. $u_{1} u_{3} u_{5} u_{7} \ldots \in W$,

2. $u_{2} u_{4} u_{6} u_{8} \ldots \in W$,

3. $(\star) \forall i\left(u_{i}\right)^{\omega} \in W$,

then $u_{1} u_{2} u_{3} u_{4} \ldots \in W$.

A winning condition $W$ is weakly concave iff its complement is weakly convex.

In the case of normal convexity there is no $(\star)$.

[GZ04] defines fairly mixing payoff mappings; in the case of prefix independent winning conditions fairly mixing resolves to the conjunction of weak concavity and weak convexity. Theorem 1 from [GZ04] says that games on finite arenas with fairly mixing payoff mappings are positionally determined.

Unfortunately, weak concavity is not enough for half-positional finite determinacy.

Proposition 2. There exists a weakly concave winning condition $W Q$ which is not half-positionally finitely determined.

Proof. Let $C=\{0,1\}$. For $w \in C^{\omega}$ let $P_{n}(w)$ be the number of 1's among the first $n$ letters of $w$, divided by $n$. The winning condition $W Q$ is a set of $w$ such that $P_{n}(w)$ is convergent and its limit is rational. It can be easily seen that for each $u \in C^{+}$we have $u^{\omega} \in W Q$. Therefore $(\star)$ is never satisfied for the complement of $W Q$, hence $W Q$ is a weakly concave winning condition. However, $W Q$ is not half-positionally determined. Consider the arena with two positions $E \in \operatorname{Pos}_{E}, A \in \operatorname{Pos}_{A}$, and moves $(E, A, 0),(E, A, 1),(A, E, 0)$ and $(A, E, 1)$. If Eve always moves in the same way, Adam can choose the moves 0 and 1 in an irrational proportion, ensuring his victory. However, Eve wins by always moving with the color opposite to Adam's last move - the limit of $P_{n}(w)$ is then $1 / 2$. 
Note that the given $W Q$ satisfies the even stronger condition obtained by replacing $\forall i$ by $\exists i$ in ( $\star$ ) in Definition 5 .

\section{Geometrical Conditions}

In this section we will show some half-positional determinacy results for geometrical conditions, which are based on the ideas similar to that used by the mean payoff game (also called Ehrenfeucht-Mycielski game). We will also show the relations between geometrical conditions and concave winning conditions.

Let $C=[0,1]^{n}$ (where $[0,1]$ is the real interval; we can also use any compact and convex subset of a normed space). For a word $w \in C^{+}$, let $P(w)$ be the average color of $w$, i.e., $\frac{1}{|w|} \sum_{k=1}^{|w|} w_{k}$. For a word $w \in C^{\omega}$, let $P_{n}(w)=P\left(w_{\mid n}\right)$ $\left(w_{\mid n}-\right.$ an $n$-letter prefix of $\left.w\right)$.

Let $A \subseteq C$. We want to construct a winning condition $W$ such that $w \in W$ whenever the limit of $P_{n}(w)$ belongs to $A$. Since not every sequence has a limit, we have to define the winner for all other sequences.

Let $W F(A)$ be a set of $w$ such that each cluster point of $P_{n}(w)$ is an element of $A$. Let $W F^{\prime}(A)$ be a set of $w$ such that at least one cluster point of $P_{n}(w)$ is an element of $A$. Note that $W F^{\prime}(A)=C^{\omega}-W F(C-A)$.

As we will see, for half-positional determinacy the important property of $A$ is whether the complement of $A$ is convex - we will call such sets $A$ co-convex (as concave usually means "non-convex" in geometry).

Geometrical conditions have a connection with the mean payoff game, whose finite positional determinacy has been proven in [EM79]. In the mean payoff game, $C$ is a segment in $\mathbb{R}$ and the payoff mapping is $u(w)=\lim _{\sup _{n \rightarrow \infty}} P_{n}(w)$ or $u(w)=\liminf _{n \rightarrow \infty} P_{n}(w)$. If $A=\left\{x: x \geq x_{0}\right\}$ then $u^{-1}(A)$ ("Eve wants $x_{0}$ or more") is exactly the geometrical condition $W F(A)$ or $W F^{\prime}(A)$. Geometrical conditions are a generalization of such winning conditions to a larger class of sets $A$ and $C$.

The following table summarizes what we know about concavity and halfpositional determinacy of geometrical conditions. In every point except No. 0 we assume that $A$ is non-trivial, i.e. $\emptyset \neq A \subsetneq C$. The first two columns specify assumptions about $A$ and whether we consider $W F(A)$ or $W F^{\prime}(A)$, and the last two answer whether the considered condition is concave and whether it has finite and/or infinite half-positional determinacy. Negative answer means that the answer is negative for all sets $A$ in the given class; the question mark means that the given problem has not been solved yet (but we suppose that the answer is positive).

\begin{tabular}{c|cc|ccc} 
No. & $A$ & condition & concavity & finite infinite \\
\hline 0 & trivial & $W F^{\prime}(A)$ or $W F(A)$ & yes & yes & yes \\
1 & not co-convex $W F^{\prime}(A)$ or $W F(A)$ & no & no & no \\
2 & co-convex & $W F^{\prime}(A)$ & yes & yes & no \\
3 & co-convex, not open & $W F(A)$ & weak only & yes? & no \\
4 & co-convex, open & $W F(A)$ & weak only & yes? & yes?
\end{tabular}


Note that, for any set $A$ which is co-convex and non-trivial, $W F^{\prime}(A)$ is finitely half-positionally determined, but not infinitely half-positionally determied. This shows a big gap between half-positional determinacy on finite and infinite arenas.

The point 4 remains open in general, but we have a positive result for a special $A$. Its proof is quite complicated; it uses a similar idea as the proof of Thm 6 below (instead of a state, we use here a real number meaning Eve's „,reserve” before falling out of $A$ ).

Theorem 5. Let $f$ be an affine function on $C$, and $A=f^{-1}(\{x \in \mathbb{R}: x<0\})$. The condition $W F(A)=\left\{w: \lim \sup P_{n}(f(w))<0\right\}$ is positional/suspendable.

Note that $W F\left(A_{1}\right) \cup W F\left(A_{2}\right)$ usually is not equal to $W F\left(A_{1} \cup A_{2}\right)$, so a union of positional/suspendable conditions given above usually is not of form $W F(A)$ itself.

\section{Monotonic Automata}

In this section we will show yet another class of half-positionally determined winning conditions. It is based on a different idea than that of concave conditions, and guarantees half-positional determinacy even for infinite arenas. We will need to introduce a special kind of deterministic finite automaton.

Definition 6. A monotonic automaton $A=(n, \sigma)$ over an alphabet $C$ is a deterministic finite automaton where:

- the set of states is $Q=\{0, \ldots, n\}$;

- the initial state is 0 , and the accepting state is $n$;

- the transition function $\sigma: Q \times C \rightarrow Q$ is monotonic in the first component, i.e., $q \leq q^{\prime}$ implies $\sigma(q, c) \leq \sigma\left(q^{\prime}, c\right)$.

Actually, we need not require that the set of states is finite. All the results presented here except for Thm 7 and the remark about finite memory of Adam can be proven with a weaker assumption that $Q$ has a minimum (initial state) and its each non-empty subset has a maximum.

The function $\sigma$ is extended to $C^{*}$ as usual: $\sigma^{*}(q, \epsilon)=q, \sigma^{*}(q, w c)=\sigma\left(\sigma^{*}(q, w), c\right)$ $\left(w \in C^{*}, c \in C\right)$. So defined $\sigma^{*}$ is still monotonic. By $L_{A}$ we denote the language accepted (recognized) by $A$, i.e., the set of words $w \in C^{*}$ such that $\sigma(0, w)=n$.

Example 4. Monotonic automata can recognize the following languages: $C^{*} a^{n} C^{*}$, $C^{*} a^{n-1} b C^{*}, C^{*} b a^{n-1} C^{*}$. Monotonic automata cannot recognize the following languages: $C^{*} a^{2} b^{2} C^{*}, C^{*} b a b C^{*}, C^{*} b a c C^{*}$.

Definition 7. A monotonic condition is a winning condition of form $W M_{A}=$ $C^{\omega}-L_{A}^{\omega}$ for some monotonic automaton $A$.

Note that if $w \in L_{A}$ then $u w \in L_{A}$ for each $u \in C^{*}$. Hence $L_{A}=C^{*} L_{A}$. Therefore $L_{A}^{\omega}$ is equal to $L_{A}\left(C^{*} L_{A}\right)^{\omega}=\left(L_{A} C^{*}\right)^{\omega}$. Hence without affecting $W M_{A}$ we can assume that $\sigma(n, c)=n$ for each $c$. 
Theorem 6. Any monotonic condition is positional/suspendable.

The proof is based on the folowing idea. We construct a new game $\left(G^{\prime}, W^{\prime}\right)$ where $\operatorname{Pos}^{\prime}=\operatorname{Pos} \times Q$, moves are natural and $W^{\prime}=C^{\omega}-L_{A} C^{\omega}$. This game is positionally determined (equivalent to $W P_{1}$ ). From monotonicity we know which position $(g, q)$ for a given $g$ is worst for Eve, and she can always play as if she were in the worst possible state. If Eve can win nowhere, then Adam wins everywhere in the original game; otherwise, Eve wins in some subset of $G$ using a positional strategy, which we can remove using Lemma 1. It is worth to remark that although Adam's strategy given in the proof is not positional, it uses only finite memory ( $Q$ is the set of memory states).

From this theorem and the examples of languages recognized by monotonic automata above one can see that e.g. $W A_{n}$, the complement of the set of words containing $a^{n}$ infinitely many times, is monotonic and thus half-positionally determined.

For $n=1$ the set $W A_{n}$ is just a co-Büchi condition. However, for $n>1$ it is easily shown that $W A_{n}$ is not (fully) positionally determined, and also that it is not concave. For example, for $n=2$ the word $(b a b a b b a b a b)^{\omega}$ is a combination of $(b b b a a)^{\omega}$ and $(a a b b b)^{\omega}$. However, all monotonic conditions are weakly concave (if $\forall_{i} w_{i}^{\omega} \in W L_{A}^{\omega}$ for $A=(n, \sigma)$, then $w_{1} w_{2} w_{3} \ldots \in W L_{A}^{\omega}$ ).

Proposition 3. Monotonic conditions are closed under finite union.

A countable union of monotonic conditions is not necessarily defined by a single monotonic automaton, but it is still positional/suspendable; however, a union of cardinality $2^{\omega}$ of monotonic conditions does not have to be half-positionally determined, since co-Büchi conditions are monotonic. Monotonic conditions are not closed under other Boolean operations.

Theorem 7. Let $W_{1} \subseteq C^{\omega}$ be a concave winning condition, and $A$ be a monotonic automaton. Then the union $W=W_{1} \cup W M_{A}$ is a half-positionally finitely determined winning condition.

\section{Conclusion and future work}

We would like to know more closure properties of the class of half-positionally determined winning conditions. Specifically we want to know whether it is closed under finite and countable union (Conjecture 1). In this paper we have proven that it is closed under union with Büchi conditions and intersection with co-Büchi conditions (Thm 1). We have also proven (Theorem 3) that positional/suspendable winning conditions are closed under countable union; and many half-positional winning conditions fall into this category. It seems worthwhile to extend Thm 3 to conditions obtained by using Thm 1 on positional/suspendable winning conditions.

Additionally, some of our results give new proofs of known facts about positional determinacy. Many previous proofs can be simplified by using Lemma 1. 
In our opinion the proof of positional determinacy of parity conditions obtained by using Thm 1 is simpler than the proofs previously known to us.

Another direction of further research is to find more examples of half-positional conditions. Theorems 1 and 3 can be used to create new half-positional conditions from old ones. They could be also obtained e.g. by generalizing the results on geometrical conditions and monotonic automata. It would be also interesting to see whether monotonic automata have applications in other areas of automata theory.

\section{References}

[BSW03] A. Bouquet, O. Serre, I. Walukiewicz, Pushdown games with unboundedness and regular conditions, Proc. of FSTTCS'03, LCNS, volume 2914, pages 88-99, 2003.

[CDT02] T. Cachat, J. Duparc, W. Thomas, Pushdown games with a $\Sigma_{3}$ winning condition. Proc. of CSL 2002, LCNS, volume 2471, pages 322-336, 2002.

[CN06] T. Colcombet, D. Niwiński, On the positional determinacy of edge-labeled games. Theor. Comput. Sci. 352 (2006), pages 190-196

[EJ91] E. A. Emerson and C. S. Jutla, Tree automata, mu-calculus and determinacy. Proceedings 32th Annual IEEE Symp. on Foundations of Comput. Sci., pages 368377. IEEE Computer Society Press, 1991.

[EM79] A. Ehrenfeucht, J. Mycielski, Positional strategies for mean payoff games. IJGT, 8:109-113, 1979.

[Gim04] H. Gimbert, Parity and Exploration Games on Infinite Graphs. Proc. of CSL '04, volume 3210 de Lect. Notes Comp. Sci., pages 56-70.

[Gra04] E. Grädel, Positional Determinacy of Infinite Games. In STACS 2004, LNCS, volume 2996, pages 4-18, 2004.

[GTW02] E. Grädel, W. Thomas, and T. Wilke, eds., Automata, Logics, and Infinite Games. No. 2500 in Lecture Notes in Compter Science, Springer-Verlag, 2002.

[GZ04] H. Gimbert, W. Zielonka, When can you play positionally? Proc. of MFCS '04, volume 3153 of Lect. Notes Comp. Sci., pages 686-697. Springer, 2004.

[GZ05] H. Gimbert, W. Zielonka, Games Where You Can Play Optimally Without Any Memory. Accepted for CONCUR 2005.

[Kla92] N. Klarlund, Progress measures, immediate determinacy, and a subset construction for tree automata. Proc. 7th IEEE Symp. on Logic in Computer Science, 1992.

[Kop06] E. Kopczyński, Half-positional determinacy of infinite games. Draft. http://www .mimuw .edu.pl/ erykk/papers/hpwc.ps

[Mar75] D. A. Martin, Borel determinacy. Ann. Math., 102:363-371, 1975.

[McN93] R. McNaughton, Infinite games played on finite graphs. Annals of Pure and Applied Logic, 65:149-184, 1993.

[Mos91] A. W. Mostowski, Games with forbidden positions. Technical Report 78, Uniwersytet Gdański, Instytut Matematyki, 1991. 